\title{
QUADRUPOLAR INTERACTIONS IN TbAg ANTIFERROMAGNETIC COMPOUND
}

\author{
R. Aléonard, P. Morin and J. Rouchy \\ Laboratoire Louis Néel, C.N.R.S., 166X, 38042 Grenoble cedex, France
}

\begin{abstract}
The strength of the quadrupolar interactions is determined by means of ultrasonic velocity experiments. For the tetragonal symmetry, the ferroquadrupolar terms are strong enough to explain the first-order transition at the Néel temperature. Their actual effects in the magnetic structure are not fully determined.
\end{abstract}

\section{Introduction}

The CsCl-type compound $\mathrm{TbAg}$ antiferromagnetically orders at $T_{\mathrm{N}}=103.5 \mathrm{~K}$. The transition is firstorder as proved by transport properties and magnetization data [1]. The jump of the magnetic moment is weak and magnetization data show the spin system to be just above tricriticallity; $\mathrm{TbAg}$ exhibits then the highest first-order magnetic transition known in rare earth intermetallics. The previous record value for CsCl-type compounds was $30 \mathrm{~K}$ in CeZn [2]. Usually for these compounds, the first-order character is driven by quadrupolar interactions, which induce an upwards initial curvature of the magnetization response to the exchange field and then a non zero value for the magnetic order parameter at the magnetic transition [3].

\section{Velocity measurements}

In order to check the validity of a quadrupolar origin for the fitst-order Néel transition in $\mathrm{TbAg}$, ultrasonic velocity measurements have been performed in single crystals. Indeed fitting the elastic softenings occurring in the paramagnetic phase usually allows one to determine both the magnetoelastic coupling and the quadrupolar pair interactions [4]. For instance in the case of the tetragonal symmetry lowering mode, the actual $C_{11}-C_{12}$ elastic constant receives besides $C_{11}^{0}-C_{12}^{0}$, the usual background behaviour without magnetic interactions, a magnetoelastic contribution:

$$
C_{11}-C_{12}=C_{11}^{0}-C_{12}^{0}-\frac{\left(B^{\gamma}\right)^{2} \chi^{\gamma}}{1-K^{\gamma} \chi^{\gamma}}
$$

$B^{\gamma}$ is the magnetoelastic coefficient, $K^{\gamma}$ the pair interaction one and $\chi^{\gamma}$ is the strain susceptibility. A similar expression exists for the trigonal-symmetry mode, $C_{44}$.

Several ultrasonic propagation modes have been studied. Figure 1 shows the temperature dependence of the $C_{11}$ elastic constant. A weak softening is observed in the paramagnetic range. A sharp anomaly occurs at $T_{\mathrm{N}}$, related to the first-order character of the transition. Echoes, although damped, exist in the ordered phase and exhibit a small jump around $29 \mathrm{~K}$.

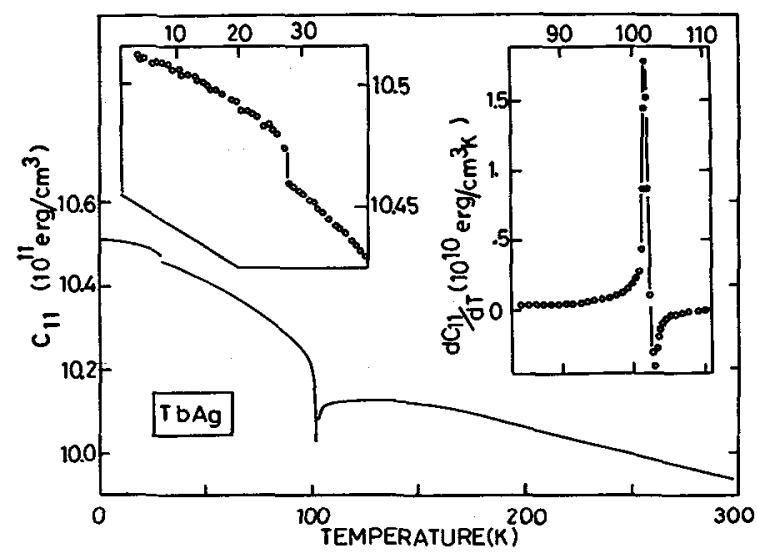

Fig. 1. - Temperature variation of $C_{11}$ in $\mathrm{TbAg}$. The insets show the anomaly at $T_{\mathrm{N}}$ and around $29 \mathrm{~K}$.

This anomaly was also observed in another ultrasonic mode $\left(C_{11}+C_{12}+2 C_{44}\right)$ which includes a $C^{\gamma}=C_{11}-$ $C_{12}$ contribution in its expansion in normal elastic modes. On the contrary, the anomaly does not exist for the trigonal symmetry normal mode $C_{44}$. The temperature dependence of $C_{11}-C_{12}$ shows a pronounced softening (Fig. 2). Echoes are lost below $T_{\mathrm{N}}$ due to a large damping associated to the magnetic domain structure. Using a similar lattice background $C_{11}^{0}-C_{12}^{0}$ as for TmAg and DyAg [4] and a strain susceptibility $\chi^{\gamma}(T)$ calculated within a crystalline electric field coherent for RAg compounds, equation (1) leads to $B^{\gamma}=24 \pm 2 \mathrm{~K}$ and $K^{\gamma}=15 \mp 1.5 \mathrm{mK}$. Thus as in other CsCl-type compounds, the quadrupolar coupling is strongly positive for the tetragonal symmetry; it is weak for the trigonal one without sizeable effects on the $C_{44}$ mode.

\section{Discussion}

The total quadrupolar coefficient receives magnetoelastic and pair coupling contributions: $G^{\gamma}=$ $\frac{\left(B^{\gamma}\right)^{2}}{C_{11}^{0}-C_{12}^{0}}+K^{\gamma}=18 \pm 2 \mathrm{mK}$. It has been shown 


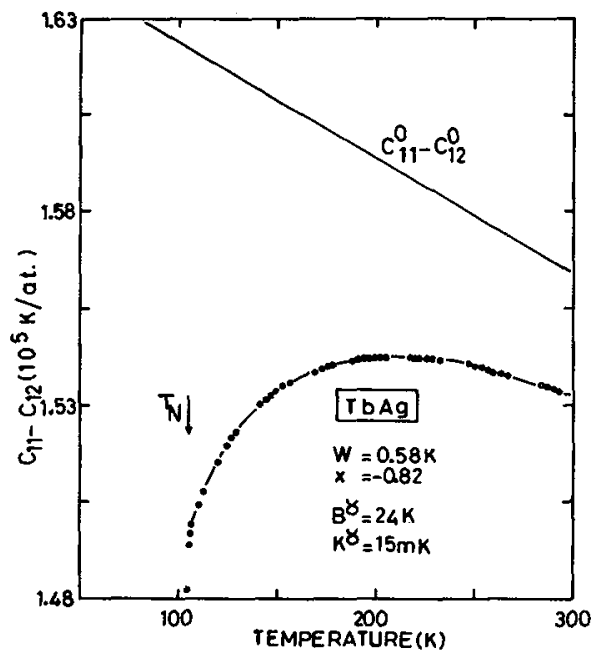

Fig. 2. - Temperature variation of $C_{11}-C_{12}$ above $T_{N}$ in TbAg. The full line is calculated with the coefficients indicated and using the $C_{11}^{0}-C_{12}^{0}$ lattice background observed in $\mathrm{YAg}$.

[3] to determine the initial curvature of the magnetization response through the third-order susceptibility $\left(M=\chi_{M}^{(1)} H+\chi_{M}^{(3)} H^{3}+\cdots\right):$

$$
\chi_{M}^{(3)} \sim\left[\chi_{0}^{(3)}+\frac{2 G^{\gamma}\left(\chi^{\gamma^{2}}\right)^{2}}{1-G^{\gamma} \chi^{\gamma}}\right]
$$

$\chi_{0}^{(3)}$ being the (usually negative) curvature of the magnetization curve in the absence of quadrupolar interactions and $\chi^{\gamma^{2}}$ a quadrupolar field-susceptibility. Due to $G^{\gamma,} \chi^{\gamma 2}, \chi_{M}^{(3)}$ may become positive at a tricritical tempeature, below which, according to Landau theory, the magnetic transition is first-order [3]. Figure 3 shows the $G^{\gamma}$-dependence of the tricritical point $T_{c}^{*}$ separating first- and second-order transitions. The experimental $G^{\gamma}$ value is strong enough to drive a firstorder transition at $T_{\mathrm{N}}=103.5 \mathrm{~K}$.

In $\mathrm{RAg}$ compounds, the nature of bilinear interactions leads to amplitude modulated spin structures described by incommensurate propagation vectors. They have been observed in all the temperature range of Tm- and HoAg [5] and close to $T_{\mathrm{N}}$ in Er- and DyAg $[6,7]$. In these latter two, due to the magnetic ground state, a commensurate structure replaces at low temperature the modulated one, which would imply zero moments at given $4 \mathrm{f}$ sites. In $\mathrm{TbAg}$, the spin modulation would be allowed to exist at $0 \mathrm{~K}$ as the $\Gamma_{3}$ ground state is non magnetic. However, this $\Gamma_{3}$ doublet has an intrinsic quadrupolar moment and similar entropy effects, but associated with modulated quadrupoles, prevent the system to keep a modulated arrangement for both spins and quadrupoles down to $0 \mathrm{~K}$. Thus, the

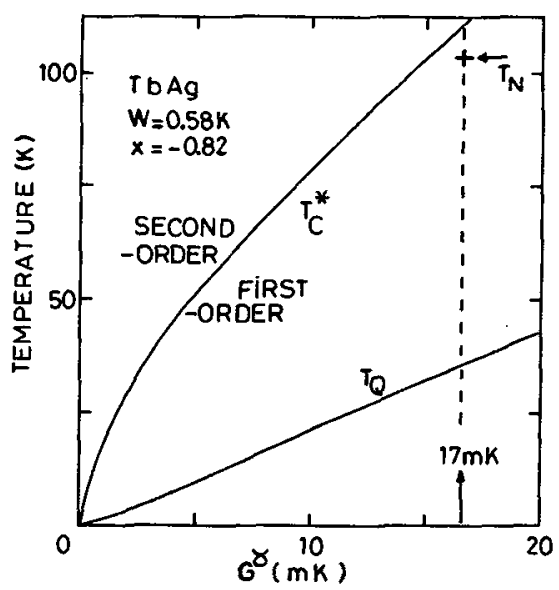

Fig. 3. $-G^{\gamma}$ dependences of the $T_{c}^{*}$ tricritical temperature and the $T_{\mathrm{Q}}$ quadrupolar ordering temperature.

system must move to a commensurate state through either a progressive squaring-up or a commensurateincommensurate transition at a given temperature depending on the strength of the ferroquadrupolax interactions. Experimentally, the (1/2 1/2 0) commensurate structure has been actually observed by neutron diffraction at $4.2 \mathrm{~K}$ in $\mathrm{TbAg}$ [8]. Unfortunately, no data are available at higher temperature in the ordered phase and the stability range of the (1/2 $1 / 20)$ phase is unknown. However one might speculate that the anomaly observed around $29 \mathrm{~K}$ on ultrasonic waves including the tetragonal symmetry mode $C^{\gamma}$ is associated to such a change of the magnetic structure. This temperature of $29 \mathrm{~K}$ is of the same order of magnitude as the quadrupolar ordering temperature which would occur with $G^{\gamma}=17 \mathrm{mK}$ in the absence of bilinear interactions (see Fig. 3). This may support the above suggestion.

[1] Morin, P., Rouchy, J., Amado,M. M., Pinto, R. P., Moreira, J. M., Amaral, V. S., Braga, M. E. and Sousa, J. B. (this conference).

[2] Pierre, J., Murani, A. P. and Galera, R. M., J. Phys. F 11 (1981) 679.

[3] Morin, P. and Schmitt, D., Phys. Rev. B 27 (1983) 4412.

[4] Giraud, M. and Morin, P., J. Magn. Magn. Mater. 58 (1986) 135.

[5] Morin, P., Schmitt, D. and Vettier, C., J. Phys. France 46 (1985) 39.

[6] Nereson, N., J. Appl. Phys. 44 (1973) 4727.

[7] Kaneko, T., Yoshida, H., Ohashi, M. and Abe, S., J. Magn. Magn. Mater. 70 (1987) 277.

[8] Cable, J., Koehler, W. C. and Wollan, E., Phys. Rev. A 136 (1964) 240. 